\title{
La Eponimia ese Difícil Obstáculo que Aún no Supera la Terminología Anatómica Internacional
}

\author{
Eponymy that Difficult Obstacle that International Anatomical Terminology still does not \\ Overcome
}

\author{
Jorge Eduardo Duque Parra ${ }^{1,2}$; John Barco Ríos ${ }^{1}$ \& Manuela Duque Quintero ${ }^{3}$
}

DUQUE, P. J. E.; BARCO, R. J. \& DUQUE, Q. M. La eponimia, ese dificil obstáculo que aun no supera la Terminología Anatómica Internacional. Int. J. Morphol., 36(4):1206-1209, 2018.

RESUMEN: El lenguaje terminológico es un instrumento que emplean las distintas ciencias para transmitir el conocimiento de manera precisa y sin ambigüedades. Debido a la enorme cantidad de términos que existían para nombrar las distintas estructuras anatómicas, en 1887 se plantea la necesidad de crear una terminología apropiada, y en 1895 surge la Nomina Anatomica de Basilea. A partir de entonces se han propuesto otras terminologías, hasta que finalmente en 1998 el Comité Federativo Internacional de Terminología Anatómica publica la actual Terminologia Anatomica. A pesar de todos los esfuerzos para tratar de unificar el lenguaje anatómico en las ciencias morfológicas, lo que mejoraría la comunicación entre los profesionales de la salud y facilitaría el proceso enseñanza-aprendizaje, aun hay mucho arraigo de parte de un sector de esta comunidad académica por la terminología tradicional dondepredominan los epónimos; mientras que otro sector desconoce la existencia de la Terminologia Anatomica o simplemente no les interesa actualizar su lenguaje terminológico. Es necesario que las nuevas generaciones de docentes de ciencias morfológicas y editores de revistas conozcan y apliquen la terminología anatómica internacional, pero que además hagan tomar consciencia a sus estudiantes que es más fácil aprender el lenguaje anatómico cuando se usa la lógica morfo-funcional, comparado con el aprendizaje memorístico de términos epónimos que no brindan ninguna información descriptiva.

PALABRAS CLAVE: Anatomía; Epónimos; Terminologia Anatomica.

\section{INTRODUCCIÓN}

La Anatomía es una de las ciencias básicas más importante que se enseña en todas escuelas de formación médica (Davis et al., 2014) de todo el mundo (Duque Parra et al., 2016). Para su estudio, se emplean términos especiales que permiten el reconocimiento y descripción de todas las estructuras que constituyen el cuerpo humano (Lippert, 2005). Esto tiende a lograrse con el uso de una terminología anatómica estándar de carácter internacional para asegurar una comunicación clara (Neumann et al., 2017) entre los estudiosos de esta ciencia. La terminología, en general, es un instrumento lingüístico especializado que emplea la ciencia para transmitir el conocimiento de manera precisa y sin ambigüedades. Es mediante la terminología que se facilita la comunicación científica y técnica para llegar a un entendimiento universal (Cabré, 2000).

En el campo anatómico, a finales del siglo XIX, se creó la necesidad de establecer un orden en su terminología, pues existían cerca de 50.000 términos anatómicos para nombrar un poco más de 5.000 estructuras corporales (Losardo et al., 2015), con una proliferación de términos para nombrar la misma estructura (Cruz et al., 2010), lo que generaba confusión y dificultad en la comunicación entre los morfólogos. Es así como en 1887 la Sociedad Alemana de Anatomistas crea el primer comité de expertos, cuya función era normatizar y unificar la terminología, asignándole en lo posible un solo nombre para cada estructura. Esta nomenclatura fue presentada y aprobada finalmente en el IX Congreso de la Anatomische Gesellschaft, celebrado en Basilea (Suiza) en 1895, y surge entonces la denominada Nomina Anatomica de Basilea (NAB) (Cruz et al.; Losardo et al.). Sin embargo, los franceses no se acogieron a dicha nómina y siguieron usando su terminología tradicional, mientras que los británicos adoptaron una nueva, conocida como la Birmingham Revision. Poco tiempo después, en 1935, la Anatomische Gesellschaft realiza una nueva revisión y aparece la denominada Jena Nomina Anatomica.

\footnotetext{
${ }^{1}$ Universidad de Caldas, Facultad de Ciencias para la Salud, Departamento de Ciencias Básicas, Manizales, Colombia.

${ }^{2}$ Universidad de Manizales, Facultad de Ciencias para la Salud, Departamento de Ciencias Básicas, Manizales, Colombia

${ }^{3}$ Universidad Autónoma de Manizales, Facultad de estudios Sociales y Empresariales, Manizales, Colombia.
} 
Durante el V Congreso Internacional de Anatomistas, realizado en Oxford en 1950, la International Federation of Association of Anatomists (IFAA) crea el Comité Internacional de Nomenclatura Anatómica (IANC), el cual tiene como tarea la revisión de la NAB y la consecuente creación de una nueva nomenclatura que pudiera ser acogida por todos los anatomistas. El fruto de este trabajo fue presentado y aprobado durante el VI Congreso Internacional de Anatomistas, celebrado en París en 1955, por lo que recibió el nombre de Nomina Anatomica de París (Cruz et al.). La IANC siguió con su trabajo de revisar continuamente la terminología, agregando algunos términos nuevos y eliminando otros tantos, y su trabajo iba siendo aprobado y publicado en los sucesivos congresos internacionales de anatomistas. Pero en 1985, durante la celebración del XII Congreso realizado en Londres, se presentaron problemas internos entre la IFAA y la IANC que ocasionaron su separación. En 1989, con la celebración del XIII congreso de anatomistas realizado en Río de Janeiro, la IFAA crea una nueva agremiación que se denomina Comité Federativo Internacional de Terminologia Anatomica (FICAT) (Hernández, 2009), encargada de seguir revisando permanentemente la terminología anatómica internacional. Finalmente, en 1998 la FICAT publica el producto de esta labor, la actual Terminologia Anatomica, y procura además que: 1) el lenguaje empleado sea el latín; 2) que casi todas las estructuras anatómicas tengan un solo nombre; 3) que los términos empleados tengan un valor descriptivo y 4) que se eliminen las eponimias, es decir el señalamiento de un detalle anatómico empleando el nombre de una persona (Cruz et al.). Con relación a este último punto, la eliminación de los epónimos se estableció formalmente con la declaración del Comité Internacional de Nomenclatura Anatómica en 1933 (Cruz \& Cruz, 2009). No obstante, la eponimia sigue muy arraigada en la actualidad en el lenguaje de las ciencias médicas, incluso es usada indebidamente por muchos profesores de anatomía y en revistas del campo morfológico, clínico y quirúrgico.

\section{DISCUSIÓN}

A pesar de la existencia de una Terminologia Anatomica, en la que se encuentran incluidos los términos que se han convenido y aceptado por la Federative International Committee on Anatomical Terminology (FICAT), con previa consulta a todos los miembros de la International Federation of Association of Anatomists (IFAA) (FICAT, 1998; Losardo et al.; Vásquez \& del Sol, 2015), aún se siguen usando de manera indiscriminada muchos términos obsoletos y epónimos para nombrar ciertas estructuras anatómicas. Pero lo más desconcertante es que muchos profesores de anatomía, de los que se supone que deberían manejar correctamente la terminología anatómica internacional (Duque Parra et al., 2016), siguen usando un lenguaje anatómico cargado de epónimos, desconociendo que el uso de ellos es un desatino en la lógica del pensamiento actual y que además fueron abolidos desde hace un poco más de ocho décadas.

El uso de epónimos y de términos obsoletos en el lenguaje médico ha generado, y sigue generando, graves problemas de comunicación entre los profesionales de la salud y también entorpece el proceso de enseñanza-aprendizaje, porque además de no brindar ninguna información descriptiva sobre unadeterminada estructura, generan errores históricos e injusticias que tienden a perpetuarse (Duque-Parra et al., 2018), y no se honra en muchos casos la memoria de quien realmente hizo el descubrimiento o la descripción inicial de una estructura anatómica.

La Terminologia Anatomica propende por una comunicación más clara y precisa entre los profesionales de las ciencias médicas, además de facilitar el aprendizaje (Duque Parra et al., 2016), porque los términos seleccionados relacionan las características morfológicas con la función (Avila et al., 2009), y también por la eliminación de múltiples términos que se tenían para una misma estructura. Sin embargo, a pesar de estos beneficios, muchos profesores, especialmente del área clínica, siguen arraigados a una terminología tradicional y obsoleta, denotando con ello su falta de actualización o interés en el campo morfológico, pero lo más grave es que menosprecian e invalidan el nuevo lenguaje anatómico que sus estudiantes adquirieron en su paso por las ciencias básicas. ¿Acaso se debería culpar también a los estudiantes que no se oponen ni hacen resistencia conceptual a estos profesores que quieren imponerles una terminología basada en epónimos? El problema es que al final terminan incorporándola también a su lenguaje, puesto que en el proceso enseñanza-aprendizaje se da un juego de persuasión, donde por lo general es el estudiante quien termina por aceptar sin mayores cuestionamientos lo que su profesor dice.

También es recurrente observar en múltiples revistas impresas del área anatómica, reconocidas mundialmente, que permiten la publicación de artículos que emplean indiscriminadamente los epónimos. Por ejemplo, en la revista The Anatomical Record se puede identificar el epónimo "Pacinian corpuscle" (Uguen, 2018); en la revista Clinical Anatomy se publica el término "vein of Galen" (Ghali, 2018); en la revista Frontiers in Neuroanatomy se incluye en uno de sus apartes el término "circle of Willis" (Qian et al., 2018); en la revista Morphologie se lee el término "Waldeyer's fascia" (Rusu et al., 2017). Estos desatinos terminológicos demuestran indudablemente que, a pe- 
sar de tantos años de trabajo y esfuerzo de la FICAT, el lenguaje anatómico sigue siendo caótico, donde cada cual habla y escribe como quiere.

Otra incongruencia terminológica involucra también a la misma FICAT. Los señores Shane Tubbs y Marious Loukas son coordinadores y asesores oficiales de la IFAA, pero además Tubbs es editor en jefe y Loukas es editor asociado de la revista Clinical Anatomy. Como miembros de la IFAA, se supone que deberían velar y promover el uso correcto de la terminología anatómica, y como editores de esa revista, deberían establecer los filtros adecuados para evitar la publicación de trabajos que no sigan los parámetros de la terminología anatómica internacional. Sin embargo, en algunos trabajos de reciente publicación en dicha revista, donde ambos figuran como coautores, se incluyen epónimos para nombrar ciertas estructuras anatómicas, como los siguientes: saco de Douglas (Ramdhan et al., 2017), ligamento de Arnold (Oakes et al., 2017a), glándulas de Bartholin (Muhleman et al., 2017), cornu Amonnis (Oakes et al., 2017b). Si partimos del supuesto de que todos los autores de un trabajo deben haberlo leído y revisado antes de someterlo a evaluación para su posible publicación, entonces surgen las siguientes preguntas: ¿sería posible que no leyeran ninguno de los trabajos donde aparece como coautores?; si los leyeron ¿por qué permitieron el uso de epónimos para nombrar estructuras anatómicas?; si como miembros de la IFAA deben dar buen ejemplo con el uso de una correcta terminología anatómica, ¿por qué en sus artículos no se refleja este buen ejemplo?; si como editores de esa prestigiosa revista, ¿por qué permiten la publicación de artículos que no respetan los preceptos establecidos para la terminología anatómica internacional?, ¿o será acaso que el comité editorial de dicha revista no realiza una buena revisión y selección imparcial de los trabajos que se publican? Finalmente, ¿acaso la IFAA es solamente un órgano burocrático que no realiza función alguna?.

En diversos congresos de anatomía de carácter nacional e internacional, donde se reúnen reconocidos expertos e investigadores en el campo morfológico, es común escuchar en los conferencistas el uso de un lenguaje anatómico cargado de epónimos, como sucedió en el pasado 18th Congress of International Federation of Associations of Anatomists, celebrado en 2014 en Beiging (China) y avalado por la FICAT (Duque Parra et al., 2017), donde se suponía que por ser un evento organizado por la IFAA se respetaría en todo sentido el uso correcto de la Terminologia Anatomica, pero no fue así. Surgen aquí otros cuestionamientos: ¿por qué se permite la presentación de ponencias orales y en poster que violan los principios generales de la terminología anatómica en relación a la eponimia?, ¿acaso el comité científico que se crea en cada congreso de ciencias morfológicas no cumple cabalmente con sus funciones de hacer un filtro en la selección de los trabajos presentados?, ¿será que los miembros que conforman estos comités científicos desconocen la terminología anatómica internacional?.

Aunque el panorama mundial parece un poco desalentador en cuanto al uso de una terminología anatómica unificada, los editores de algunas revistas como International Journal of Morphology, Journal of Anatomy, Anatomy and Cell Biology, Journal of Morphological Sciences, entre otras, realizan una labor esperanzadora, porque además de difundir la investigación en el campo de las ciencias morfológicas que se genera en distintas latitudes, también velan por el uso de un lenguaje anatómico correcto que sigue los preceptos de la Terminologia Anatomica. Pero también es necesario que los nuevos docentes de las ciencias morfológicas (anatomía, histología y embriología -morfología de la vida intrauterina-) conozcan y hablen con propiedad el lenguaje de la terminología anatómica internacional, para que desde el aula de clase motiven y estimulen el uso correcto de este lenguaje a las nuevas generaciones de estudiantes de las ciencias médicas. Que además les haga tomar consciencia que es mucho más fácil apropiarse de un lenguaje técnico cuando se hace uso de una lógica morfo-funcional, comparado con el aprendizaje de aquel otro lenguaje morfológico tradicional que implica un mayor esfuerzo memorístico, porque hace uso alegórico de personajes históricos que no brindan ninguna información descriptiva ni funcional de lo que se está estudiando. Aunque es importante preservar los epónimos para tener una memoria histórica de aquellos personajes que hicieron grandes aportes al conocimiento morfológico, tales términos solo deberían ser empleados en otro tipo de publicaciones de carácter histórico (Abdala, 2007) o en diccionarios de regionalismos (Cruz et al.), pero no para la descripción anatómica.

\section{CONCLUSIÓN}

Aunque han transcurrido más de 120 años de haberse creado la Nomina Anatomica de Basilea como primer intento para unificar el lenguaje morfológico, a pesar de los esfuerzos realizados por la FICAT para seguir revisando y actualizando la terminología anatómica internacional y de los múltiples beneficios que traería para los estudiantes y profesionales de la salud su aplicación como único lenguaje universal, todavía existe un importante sector de esta comunidad académica que sigue empecinada en el uso de un lenguaje tradicional cargado de epónimos que solo genera confusión y entorpece la comunicación. Se espera que las nuevas generaciones de docentes de ciencias morfológicas, apo- 
yados por los editores de revistas, apliquen la terminología anatómica internacional, y que además hagan tomar consciencia a sus estudiantes que es más fácil aprender esta ciencia cuando se usa la lógica morfo-funcional.

DUQUE, P. J. E.; BARCO, R. J. \& DUQUE, Q. M. Eponymy that difficult obstacle that international anatomical terminology still does not overcome. Int. J. Morphol., 36(4):1206-1209, 2018.

SUMMARY: Language terminology is an instrument used by different sciences to transmit knowledge in a precise and unambiguous way. Due to the enormous amount of terms that existed to name the different anatomical structures, in 1887 the need arose to create an appropriate terminology, and in 1895 the Anatomical Nomina of Basel was established. Since then other terminologies have been proposed, until finally in 1998 the International Federative Committee of Anatomical Terminology publishes the current Terminologia Anatomica. Despite much effort to try to unify anatomical language in the morphological sciences, which would improve communication between health professionals and facilitate the teaching-learning process, part of the academic community continues to hold steadfast to traditional terminology, where eponyms predominate; while another sector ignores the existence of the Terminologia Anatomica, or simply does not care about updating its terminological language. It is therefore, necessary that new generations of professors of morphological sciences and journal editors know and apply the international anatomical terminology, but also make their students aware that it is easier to learn the anatomical language when using the morphofunctional logic, compared to the rote learning of eponymous terms, that does not provide any descriptive information.

KEY WORDS: Anatomy; Eponymous; Anatomical terminology.

\section{REFERENCIAS BIBLIOGRÁFICAS}

Abdala, P. Epónimos neuroanatómicos en Revista de Neurología en línea. Rev. Neurol., 45(4):201-4, 2007.

Avila, R. E.; Samar, M. E.; Acevedo, R. \& Barioglio, C. La célula vegetal como instrumento didáctico de comprensión de la mitosis en la célula animal. Arch. Anat. Costa Rica, 4:51, 2009.

Cabré, M. T. Terminologie et linguistique: la théorie des portes. Terminol. Nouv, 21:10-15, 2000.

Cruz, G. R. \& Cruz, C. F. Sugerencias para establecer un diccionario de epónimos anatómicos, embriológicos e histológicos. Arch. Anat. Costa Rica, 4:28, 2009.

Cruz, G. R.; Rodríguez, T. A.; Prates, J. C.; Losardo, J. R. \& Barbato, V. N. Ibero-Latin-American Symposia Terminology. Anatomy, Histology and Embryology. Int. J. Morphol, 28(1):333-6, 2010.

Davis, C. R.; Bates, A. S.; Ellis, H. \& Roberts, A. M. Human anatomy: let the students tell us how to teach. Anat. Sci. Educ., 7(4):262-72, 2014.

Duque Parra, J. E.; Barco Ríos, J. \& Aldana Rueda, J.E. La Terminología Anatómica en Colombia y el uso de epónimos en la enseñanza en medicina. Rev. Biosalud, 15(1):82-86, 2016.

Duque Parra, J. E.; Barco Ríos, J.; Vélez García, J. F. Eponyms prevalence in publications and presentations at the 18th Congress of International Federation of Associations of Anatomists 2014. Int. J. Morphol., 35(2):525-8, 2017.

Duque-Parra, J. E.; Barco-Ríos, J. \& Dávila-Alzate, N. Los epónimos en las ciencias médicas: errores históricos que originan injusticias. Rev. Fac. Med., 66(1):87-90, 2018.

Federative International Committee on Anatomical Terminologies (FICAT). Terminologia Anatomica. International Anatomical Terminology. New York, Thieme, 1998.

Ghali, M. G. Z. Galenic pial arteriovenous fistulas: Angioarchitecture, clinical presentation, and therapeutic considerations. Clin. Anat., 31(2):259-68, 2018.

Hernández, R.F. Raíces del Simposio Iberolatinoamericano de Terminología. Arch. Anat. Costa Rica, 4:27, 2009.

Lippert, H. Anatomía. Estructura y Morfología del Cuerpo Humano. Madrid, Marban, 2005.

Losardo, R. J.; Prates, N. E. V. B.; Arteaga-Martínez, M.; Cabral, R. H. \& García-Peláez, M. I. International Morphological Terminology: More than anatomy, histology and embryology. Int. J. Morphol, 33(1):4007, 2015.

Muhleman, M. A.; Aly, I.; Walters, A.; Topale, N.; Tubbs, R. S. \& Loukas, M. To cut or not to cut, that is the question: A review of the anatomy, the technique, risks, and benefits of an episiotomy. Clin. Anat., 30(3):362-72, 2017.

Neumann, P. E.; Baud, R. \& Sprumont, P. Human anatomy nomenclature rules for the computer age. Clin. Anat., 30(3):300-2, 2017.

Oakes, P. C.; Sardi, J. P.; Iwanaga, J.; Topale, N.; Oskouian, R. J. \& Tubbs, R. S. Translation of Hecker's 1922 "the occipital-atlanto-axial ligament system": A study in comparative anatomy. Clin. Anat., 30(3):322-9, $2017 \mathrm{~b}$.

Oakes, P.; Loukas, M.; Oskouian, R. J. \& Tubbs, R. S. The neuroanatomy of depression: A review. Clin. Anat., 30(1):44-9, 2017a.

Qian, B.; Rudy, R. F.; Cai, T. \& Du, R. Cerebral artery diameter in inbred mice varies as a function of strain. Front. Neuroanat., 12:10, 2018.

Ramdhan, R. C.; Loukas, M. \& Tubbs, R. S. Anatomical complications of hysterectomy: A review. Clin. Anat., 30(7):946-52, 2017.

Rusu, M. C.; Pop, F.; Petre, N. \& Dobra, M. A. The sheath of Waldeyer is not a specific anatomical trait of the ureterovesical junction. Morphologie, 102(336):6-11, 2017.

Uguen, A. Another case of pacinian corpuscle in a lymph node. Anat. Rec. (Hoboken), 301(4):561-2, 2018.

Vásquez, B. \& del Sol, M. Terminologia Anatomica and Terminologia Histologica. A meeting point between morphologists. Int. J. Morphol, 33(4):1585-90, 2015.

\section{Dirección para correspondencia: \\ Jorge Eduardo Duque Parra \\ Programa de Medicina \\ Departamento de Ciencias Básicas \\ Universidad de Caldas \\ Calle 65 No 26-10 \\ Manizales \\ COLOMBIA}

Email: jorge.duque_p@ucaldas.edu.co

Recibido : 29-04-2018

Aceptado: 23-07-2018 